\title{
POLYCYTHAEMIA RUBRA VERA WITH INCISIONAL HERNIA SURGERY-A VERY RARE CASE REPORT
}

\author{
KHAN MMH ${ }^{1}$, SULTANA ${ }^{2}$, RAHMAN MF ${ }^{3}$, AHSAN T ${ }^{4}$, ALAM AHMT ${ }^{5}$, HOSSAIN MN ${ }^{6}$

\begin{abstract}
:
Polycythaemia rubra vera is a chronic myeloproliferative disorder that is characterized by excessive red cell production and unlike other forms of polycythaemia, it can cause both bleeding and thrombosis in the same patient. Only few cases have been reported in the literature where polycythaemia rubra vera with surgery was performed successfully. The ideal peri-operative management is currently unknown. Here we present a case of 50 yrs old lady with polycythaemia rubra vera underwent open mesh repair for incisional hernia. After operation, the patient developed hemorrhagic complications needing resuscitation with blood and plasma expander. The patient was managed efficiently. Considering its very rarity, we are reporting the case.
\end{abstract}

Key words: Polycythaemia vera (PV).

J Dhaka Med Coll. 2018; 27(2) : 218-222

\section{Introduction:}

Polycythemia vera (PV) is presented by both spontaneous thrombosis and hemorrhage ${ }^{1}$. In general, the thrombotic tendency has been more discussed and relatively little emphasis has been placed on the hemorrhagic aspect. Burris and Arrowsmith have recently published their experience with surgery on 68 patients with $\mathrm{PV}^{2}$. This is the only report in the recent surgical literature which has discussed this problem. They observed the thrombotic tendency to be the most common postoperative complications and did not find any instance of hemorrhagic events. The purpose of this paper is to present a case of PV in which the postoperative course was complicated by hemorrhage and to suggest a more comprehensive preoperative evaluation and optimization which would help to prevent both hemorrhagic and thrombotic complications.
Primary polycythemia (polycythemia vera) is considered as a hematopoeitic stem cell disorder giving rise to proliferation of a clone of hematopoietic precursors leading to an excess production of erythrocytes with thrombocytosis and leucocytosis in some patients. Though the incidence of complications that are associated with polycythemia vera (PV) is found low, the risk of perioperative morbidity and mortality is found to be substantial if complications occur. Attention has always been on other hematological disorders, especially anemia inspite of lack of serious complications associated with anemia in perioperative period. Shift of attention towards less frequently encountered bleeding disorders such as PV should draw attention of not only the anesthesiologist but also the physicians and surgeons directly concerned with the patient. Normal stem cells are found in the bone marrow of patients with PV besides abnormal clonal

1. Dr. Md. Manir Hossain Khan, Associate Professor, Department of Surgery, Bangabandhu Sheikh Mujib Medical University (BSMMU)

2. Dr. Jobaida Sultana, Associate Professor, Department of Gynaecology and Obstetrics, Shahid Suhrawardy Medical College Hospital (ShSMC)

3. Dr. Md. Fazlur Rahma, nResident, Department of Surgery, Bangabandhu Sheikh Mujib Medical University (BSMMU)

4. Dr. Tazin Ahsan, Honorary Medical Officer, Department of Surgery, Bangabandhu Sheikh Mujib Medical University (BSMMU)

5. Dr. A.H.M. Towhidul Alam, Professor, Department of Surgery, Bangabandhu Sheikh Mujib Medical University (BSMMU)

6. Dr. Md. Nabir Hossain, Associate Professor, Department of Surgical Oncology, National Institute of Cancer Research and Hospital , Dhaka

Correspondence: Dr. Md. Manir Hossain Khan, Associate Professor, Department of Surgery, Room no- 915, Block- C, Bangabandhu Sheikh Mujib Medical University (BSMMU), Shahbagh, Dhaka-1000, Contact Number : +8801715024896, e-mail -dmanirhk@gmail.com

https://doi.org/10.3329/jdmc.v27i2.45838 
stem cells which interfere with or suppress normal stem cell growth and maturation. The origin of the stem cell transformation remains unknown. Several studies suggest that a mutation on the Janus kinase-2 gene (JAK2) is the most likely candidate gene involved in PV pathogenesis, as JAK2 is directly involved in the intracellular signaling following exposure to cytokines to which PV progenitor cells display hypersensitivity ${ }^{3}$.

Polycythemia can be divided in two groups: PV (PV primary or true) and non vera types. PV is considered as a disorder of the blood-producing cells of bone marrow which results in overproduction of red blood cells. In polycythemia vera, the extra mass of red blood cells increases the volume of blood and makes it more viscous, as a result it flows less easily through small blood vessels. The cause is unknown. Non Vera types are further sub-divied into secondary and relative polycythemia Secondary are usually caused by oxygen deprivation, as seen in living at high altitudes, smoking, chronic pulmonary disease and cyanotic heart disease. Relative polycythemia occurs mainly as a result of dehydration that is caused by the use of diuretics, or, drinking too little fluids or sweating a lot. All the non vera types have underlying causes and are not true $\mathrm{PV}^{3}$. Though the incidence of PV is not constant worldwide, approximately 1.9 in 100,000 (person each year) are diagnosed each year. It can occur in all age groups though incidence goes up with age ${ }^{3}$.

\section{Case report :}

A 50 yrs old lady coming from Norshingdi admitted into BSMMU on 12 July 2016 for open mesh repair. She has been also suffering from PV for last $1 \mathrm{yr}$ and was on medical therapy and regular phlebotomy. Her past history included hypertension and carrier of hepatitis$\mathrm{C}$ virus for last 2 yrs without any hepatic complications. She had a history of open total abdominal hysterectomy 2 yrs ago without any significant events. She had no history of smoking, COPD, heart disease. She is a housewife. Her weight is $50 \mathrm{~kg}$ and height is $162 \mathrm{~cm}$.
On examination, She was of average body built, having plethoric face and conjuctival injection. She had an overall dusky cyanotic complexion. Her pulse was 60/min and BP $-120 / 80 \mathrm{mmHg}$. Splenomegaly was found $3 \mathrm{~cm}$ from left costal margin along its long axis. A slightly tender spherical swelling present in right iliac fossa at the right end of previous pfannestial incision scar which was reducible and gap about $3 \times 4$ $\mathrm{cm}$.Pre-operative investigation findings are listed in Table I.

Table-I

Preoperative investigation profile

\begin{tabular}{lcc}
\hline Lab variable & Value & Normal range \\
\hline $\mathrm{Hb}$ & $17.2 \mathrm{gm} / \mathrm{dl}$ & $11-15 \mathrm{gm} / \mathrm{dl}$ \\
WBC & $13 \times 10^{9} / 1$ & $4-11 \times 10^{9} / 1$ \\
Platelet count & $350 \times 10^{9} / 1$ & $150-450 \times 10^{9} / 1$ \\
ESR & $34 \mathrm{~mm}$ in & Up to $20 \mathrm{~mm}$ \\
& $1 \mathrm{st} \mathrm{hour}$ & in $1 \mathrm{st} \mathrm{hour}$ \\
Hct & $79 \%$ & $35-45 \%$ \\
PT & $14 \mathrm{sec}$ & $12-14 \mathrm{sec}$ \\
INR & 1.1 & $0.8-1.2$ \\
APTT & $32 \mathrm{sec}$ & $32-35 \mathrm{sec}$ \\
Bleeding time & $03 \mathrm{~min}$ & $2-7 \mathrm{~min}$ \\
Cloting time & $83 \mathrm{u} / 1$ & $5-11 \mathrm{~min}$ \\
Albumin & $39 \mathrm{gm} / 1$ & $30-35 \mathrm{gm} / \mathrm{dl}$ \\
Alkaline phosphatase05 min & $15-65 \mathrm{u} / 1$ \\
SGPT & $23 \mathrm{u} / 1$ & $30-60 \mathrm{u} / 1$ \\
SGOT & $30 \mathrm{u} / 1$ & $30-60 \mathrm{u} / 1$ \\
HBsAg & Negative \\
AntiHcv & Positive \\
PCR for HCV RNA Undetectable \\
S.Creatinine & $0.76 \mathrm{mg} / \mathrm{dl}$ & $0.6-1.2 \mathrm{mg} / \mathrm{dl}$ \\
RBS & $5.5 \mathrm{mmol} / 1$ \\
USG of W/A & Splenomegaly \\
JAK-2 & Positive \\
\hline
\end{tabular}

After proper pre-operative optimization, she underwent open prolene mesh repair by on-lay position technique. Considerable attention was paid to meticulous technique and haemostsis. There was no preoperative adverse events. 


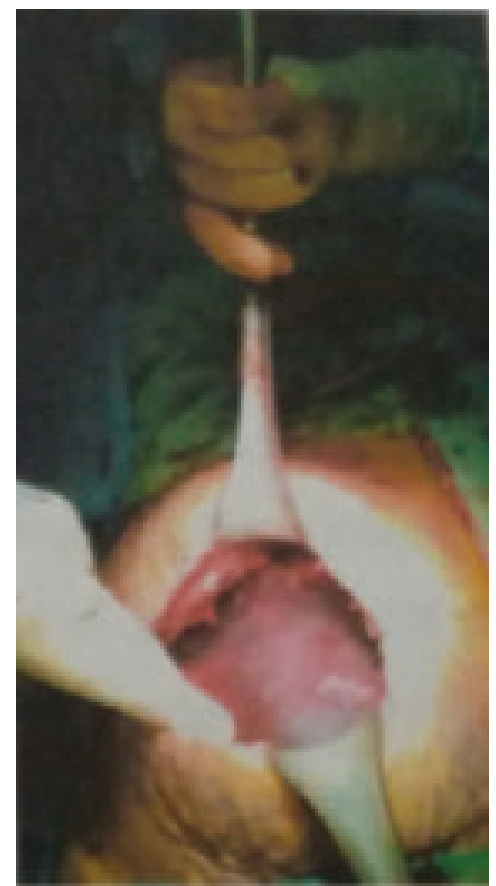

Fig.-1: During hernia surgery

Immediate recovery room, patient's vital sign was within normal limit but drain tube collection was $100 \mathrm{ml}$ ( 2 hours after operation) which was fresh blood and about $1400 \mathrm{ml}$ blood drained on "O" POD (postoperative day).We consult with hematologist. Two unit fresh blood transfused and blood sent for coagulation profile. Laboratory exam revealed coagulation profile including bleeding time, clotting time, prothombin time, APTT, FDP, D-dimer, plasma fibrinogen and von Willebrand factor were normal but it was noted that clot did not retract and easily dispersed.

On 1st and 2nd POD, fresh blood was seen through drain tube that measured about 1200 $\mathrm{ml}$ and $800 \mathrm{ml}$ respectively. On $4^{\text {th }} \mathrm{POD}$, drain tube collection was nil but haematoma formed beneath subcutaneous layer. On $7^{\text {th }}$ POD, she was taken to operation theatre and clot was evacuated under general anaesthesia. On careful inspection, no bleeding point could be found. After $2^{\text {nd }}$ time exploration, patient was stable, minor sero-sanguinous fluid drained through drain tube but uneventful recovery.

\section{Discussion}

There are several identified causes of morbidity and mortality associated with PV. Thrombosis is seen in $15-60 \%$ of patients, depending on control of the disease. It is the principal reason of death in $10-40 \%$ of patients. Venous thromboses have caused pulmonary emboli, renal failure from renal vein or renal artery thrombosis, intestinal ischemia resulting from mesenteric vascular thromboses, or peripheral arterial emboli. Hemorrhagic complications occur in $15-35 \%$ of patients and result in death in $6-30 \%$ of these patients. Peptic ulcer disease is found in association to PV at a 3- to 5-fold higher rate in compared to the general population. This has been due to increased histamine serum levels. Myelofibrosis and pancytopenia may occur in $3-10 \%$ of patients, usually in late stage of disease, which can be considered as the spent phase of PV. In these cases, bleeding and infectious complications may become the most serious health threats, and red cell transfusion may be required to maintain adequate red blood cell counts and to improve fatigue and other anemia-related symptoms. Acute leukemia or a myelodysplastic syndrome develops in $1.5 \%$ of patients who are treated with phlebotomy alone. The risk of transformation rises up to $13.5 \%$ in 5 years with treatment using chlorambucil and up to $10.2 \%$ within 6-10 years in patients treated with P-32 (radioactive Phosphorus- 32). At 15 years, transformation risk for Hydroxyurea (HU) is $5.9 \%$, which, although not statistically significant, is worrisome ${ }^{3}$.

Diagnostic criteria by polycythaemia vera study group are as follows - Category A ( total red blood cell mass - in males, more than or equal to $36 \mathrm{~mL} / \mathrm{kg}$ and in females, greater than or equal to $32 \mathrm{~mL} / \mathrm{kg}$, arterial blood oxygen saturation greater or equal to $92 \%$ and splenomegaly) and Category B (thrombocytosis with platelet count greater than 400,000/iL, leukocytosis with a white blood cell count greater than $12,000 / \mathrm{iL}$, increased leukocyte alkaline phosphatase (ALP) greater than $100 \mathrm{U} /$ $\mathrm{L}$, serum vitamin B-12 concentration more than $900 \mathrm{pg} / \mathrm{mL}$ or binding capacity more than 2200 $\mathrm{pg} / \mathrm{mL}$ ). The diagnosis is established with A1 plus A2 plus A3 or A1 plus A2 plus any 2 criteria from category-B. With the establishment of polymerase chain reaction (PCR)-based methods for detecting the JAK2 V617F mutation, this 
may become the first molecular diagnostic marker for $\mathrm{PV}^{3}$.

Venesection of $300-500 \mathrm{~mL}$ is performed weekly or twice weekly to achieve a haematocrit of less than 0.45 , and thereafter every 3-6 months to maintain the haematocrit at this level. Iron deficiency may occur and requires cautious treatment. Low-dose aspirin (100 mg/day) reduces thrombotic complications. Cytoreductive therapy should be considered when venesection is poorly tolerated. There is symptomatic or progressive splenomegaly, thrombocytosis or the presence of symptoms that may indicate disease progression (e.g. night sweats; weight loss) ${ }^{4,5}$.

It is preferred to maintain blood results at reference range levels by regular evaluation and treatment. Fasting for prolonged time without adequate replacement should be avoided as this can lead to dangerously high hematocrit levels in patients already predisposed to hypercoagulability. Fasting, even for not more than 4-6 hrs should always be accompanied by adequate pre operative hydration. During operation the patients' baseline hypercoagulability rises by a hundred fold. Aggressive hydration should be continued throughout the surgery and adequate urine output must be ensured. Emergent surgery may require intraoperative phlebotomies. This must be done with extreme caution taking care to replace lost volume and avoiding vasoconstriction from volume depletion that can risk multiorgan ischemia or intraoperative thrombus formation $6,7,8,9$.

Monitoring using central venous line is advised in all patients to monitor not only fluid status but also for the provision of a large gauge central venous line for rapid infusion rates that may be required in such patients ${ }^{10,11}$. Monitoring the vitals and clinical evaluation of patient for the possibility of stroke or hemorrhage should continue from the intraoperative through the postoperative period ${ }^{7,12,13}$.

All patients with PV who present for surgery, either elective or emergency, are advised to be admitted to the intensive care unit in the immediate post operative period for minimum forty eight hours with strict vitals observation and concurrent clinical and laboratory evaluation ${ }^{14,15}$. Early ambulation and aggressive use of analgesics preferably opioids is recommended to prevent stasis of blood flow and discourage formation of deep venous thrombosis to which these patients are at increased risk. Compression stockings to avoid clot formation and use of peripheral local anesthetic blocks to relieve postoperative pain and thus allow early ambulation should be undertaken in these patients ${ }^{3,16}$.

\section{Conclusion}

Patient's diagnosed with PV are at increased risk of perioperative thrombosis and hemorrhage. Follow-up in the post-operative period may be complicated by pulmonary or cerebral thrombosis or embolisms or hemorrhage. It might be concluded that PV is a risk factor for haemorrhagic events that requires meticulous attention to perioperative assessment of patient blood profile and subsequently tailored treatment strategies in order to avoid substantial morbidity and mortality.

\section{Reference(s)}

1. Passamonti F, Malabarba L, Orlandi E, et al.(2003). Polycythemia vera in young patients: a study on the long-term risk of thrombosis, myelofibrosis and leukemia. Haematologica 88 (1): 13-8.

2. Berlin NI (1975). Diagnosis and classification of polycythemia. Semin Hematol 12: 339.

3. Khan FA, Khan RA, Iqbal M, Hussain S. Polycythemia vera: Essential management protocols. Anaesth Pain \& Intensive Care 2012;16(1):91-9

4. Ruggeri M, Rodeghiero F, Tosetto A. Post surgery outcomes in patients with polycythemia vera and essential thrombocythemia: a retrospective survey. Blood 2008 Jan 15;111(2):666-71.

5. Weinfeld A, Swolin B, Westin J. Acute leukaemia after hydroxyurea therapy in polycythaemia vera and allied disorders: prospective study of efficacy and leukaemogenicity with therapeutic implications. Eur $J$ Haematol 1994;52(3):134-9.

6. Fruchtman SM, Pettit RM, Gilbert HS, et al, and the Anagrelide Study Group. Anagrelide: analysis of longterm safety and leukemogenic potential in myeloproliferative diseases (MPDs) [abstract]. Blood 2002;100:70a.

7. Fruchtman SM, Petitt RM, Gilbert HS, Fiddler G, Lyne $A$, and the Anagrelide Study Group Leukemia 
Research. Anagrelide: analysis of long-term efficacy, safety and leukemogenic potential in myeloproliferative disorders. Leuk Res. 2005;29(5): 481-91.

8. Landolfi R, Marchioli R, Kutti J, et al. Efficacy and safety of low-dose aspirin in polycythemia vera. $N$ Engl J Med. 2004;350(2):114-24.

9. Squizzato A, Romualdi E, Middeldorp S. Antiplatelet drugs for polycythaemia vera and essential thrombocythaemia. Cochrane database sys rev. 2008 Apr 16;(2):CD006503.

10. Dan K, Yamada T, Kimura Y Clinical features of polycythemia vera and essential thrombocythemia in Japan: retrospective analysis of a nationwide survey by the Japanese Elderly Leukemia and Lymphoma Study Group. Int $J$ Heamatol 2006;83(5):443-9.

11. Fruchtman SM, Mack K, Kaplan ME, et al. From efficacy to safety: a Polycythemia Vera Study Group report on hydroxyurea in patients with polycythemia vera. Semin Hematol 1997;34(1):17-23.
12. Berk PD, Goldberg JD, Donovan PB, et al. Therapeutic recommendations in polycythemia vera based on Polycythemia Vera Study Group protocols. Semin Hematol 1986;23(2):132-43.

13. Landolfi R, Cipriani MC, Novarese L. Thrombosis and bleeding in polycythemia vera and essential thrombocythemia: pathogenetic mechanisms and prevention. Best pract clin res heamatol. 2006; 19(3):617-33.

14. Elliott MA, Pardanani A, Lasho TL. Thrombosis in myelofibrosis: prior thrombosis is the only predictive factor and most venous events are provoked. Heamatologica 2010;95(10):1788-91.

15. De Stefano V, Za T, Rossi E. Recurrent thrombosis in patients with polycythemia vera and essential thrombocythemia: incidence, risk factors, and effect of treatments. Heamatologica 2008;93(3):372-80.

16. Beer PA, Erber WN, Campbell PJ. How I treat essential thrombocythemia. Blood 2011;117(5):1472-82. 